\title{
Design Optimization of Engine Room of Ship with Dynamic Response Using Genetic Algorithms
}

\author{
by Mitsuru Kitamura*, Member \\ Hisashi Nobukawa*, Member
}

Fengxiang Yang**, Student Member

\begin{abstract}
In this paper, the dynamic response optimization of engine room structure of ship is carried out by using the Genetic Algorithms (GAs). The static stresses and dynamic response to the external excitation are included into the constraint conditions. To save the computing storage, the floating point representation of solution in GAs is used. The operators of arithmetical crossover, boundary mutation and non-uniform mutation are introduced to improve the convergence of the GA. The effects of them are investigated. Finite element model for the static and dynamic analyses is employed in developing the Genetic Algorithm for the optimization of structural system. Modal superposition principle is used to calculate the response of the structure to the propeller excitation. The optimum results for the numerical example are presented and it shows that the GA for the dynamic response optimization is applicable.
\end{abstract}

\section{Introduction}

It is well known that giving consideration to vibration behavior in ship design is important from the safety and noise standpoints ${ }^{6}$. Reference [6] provided the optimization of engine room considering the stress and frequency constraints in which the frequency constraints are introduced to avoid the resonance of the structure to the external excitation. However, even though the natural frequencies are not in the forbidden band of frequencies, the level of vibration at some parts of structure may be larger than the allowable values. It is difficult to know how far away the natural frequencies of the structure should be from the excitation ones. In fact, the important consideration for the vibration is to control the accelerations and displacements of nodal points. Therefore, for designing the structures like engine room, it is appropriate to calculate directly the static stresses and dynamic response to keep them below the allowable values.

For general external excitations, the accelerations of nodes are dependent on time. Since all the constraints must be satisfied during the dynamic loading, this kind of the problems becomes very expensive for the optimization calculation. Until now, a minimum number of literature exists on this kind of problems and the most of it is limited to harmonic excitation. In this study,

* Faculty of Engineering, Hiroshima University

** Graduate School, Hiroshima University

Received 8th Jan. 1999

Read at the Spring meeting 12, 13th May 1999 since the vibration of the engine room is caused mainly by the harmonic propeller excitation, we take this force as the external excitation. For this computing model, the maximal values of vibration can be calculated explicitly and they are independent of time. This handling can save copious amount of computation time. Finite element model is employed for the static and dynamic analyses. The mode superposition principle is used to calculate the dynamic response. The subspace iteration method is utilized for eigenvalue analysis in the selected sample problem.

In reference [6], the authors have investigated the applicability of GAs with the binary representation technique. It is realized that the binary representation is impractical. This method requires very large computing storage when the number of design variables becomes larger and the precision of them becomes higher. As a matter of fact, the floating point representation technique is more natural and works as well as, or even better than the binary representation technique according to the recent research ${ }^{3)}$. Until recent study, there is a trend to apply the floating point representation to real problems ${ }^{3,77,8), 9}$. On the other hand, some new operators ${ }^{3), 8,9)}$ based on the real coding technique have been proposed by several researchers. Therefore in this study, the floating point representation technique is applied to the string and the effects of introducing the operators such as simple crossover, arithmetical crossover, uniform mutation, boundary mutation, and nonuniform mutation are investigated.

\section{Illustration of Problem}

The objective in this problem is to find the design 
variables $\boldsymbol{X}$ to minimize the cost of structure under the static and dynamic constraints as the following:

$\min f(\boldsymbol{X})$

subject to :

(1) Stress constraints

$\left|\sigma_{i, \max }^{s}\right|+\left|\sigma_{i, \max }^{d}\right| \leq \sigma_{i, 0} \quad i=1,2, \cdots, n e$

where the $\sigma_{i, \max }^{S}$ and $\sigma_{i, \max }^{d}$ are the extreme values of the static and dynamic stress of element $i$ respectively. $\sigma_{i, 0}$ is the allowable stress for element $i$.

(2) Displacement constraints

$\left|Y_{j}\right|+\left|U_{j}\right| \leq D_{j, 0} \quad j=1,2, \cdots, n j$

where $Y_{j}$ and $U_{j}$ are the extreme dynamic and static displacements of node $j$ respectively. $D_{j, 0}$ is the allowable displacement for node $j$.

(3) Acceleration constraints

$$
\left|\bar{a}_{j}\right| \leq a_{j, 0} \quad j=1,2, \cdots, n j
$$

where $\bar{a}_{j}$ and $a_{j, 0}$ are the maximal acceleration and its allowable value of node $j$ respectively.

(4) Design variable constraints

$x_{i, \min } \leq x_{i} \leq x_{i, \max } \quad i=1,2, \cdots, q$

The extreme static stresses in each element and the static displacement of every node can be calculated by solving the static equilibrium equation as below.

$$
\boldsymbol{K} \boldsymbol{Y}=\boldsymbol{P}
$$

The extreme dynamic stress in each element, and the dynamic displacements and acceleration of nodes can be calculated through the dynamic analysis described below.

\section{Dynamic Analysis}

The differential equations of motion for the engine room structure under the excitation $\boldsymbol{F}(t)$ can be written as below:

$$
\boldsymbol{M} \ddot{\boldsymbol{U}}+\boldsymbol{C} \dot{\boldsymbol{U}}+\boldsymbol{K} \boldsymbol{U}=\boldsymbol{F}(t)
$$

where $\boldsymbol{M}, \boldsymbol{C}, \boldsymbol{K}$ are the total mass, damping and stiffness matrix respectively. The matrices are $n \times n$, where $n$ is the number of degrees of freedom of the structure. The damping is assumed to be proportional to the stiffness and mass of structure. Based on these simplifications, the equations can be solved by modal superposition principle as the following:

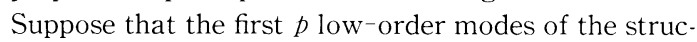
ture are represented by mode-shape matrix $\boldsymbol{\Phi}$, and are obtained by solving the frequency problem. The following coordinate transformation is introduced.

$$
\boldsymbol{U}=\boldsymbol{\Phi}_{\boldsymbol{q}}
$$

Eq. 7 can be transformed into $p$ uncoupled differential equations as

$$
\begin{aligned}
& \boldsymbol{M}^{*} \ddot{\boldsymbol{q}}+\boldsymbol{C}^{*} \dot{\boldsymbol{q}}+\boldsymbol{K}^{*} \boldsymbol{q}=\boldsymbol{F}^{*}(t) \\
& \text { where } \\
& \boldsymbol{M}^{*}=\boldsymbol{\Phi}^{T} \boldsymbol{M} \boldsymbol{\Phi}=\boldsymbol{I} \\
& \boldsymbol{C}^{*}=\boldsymbol{\Phi}^{T} \boldsymbol{C} \boldsymbol{\Phi}=\operatorname{diag}\left[2 \xi_{i} \omega_{i}\right] \\
& \boldsymbol{K}^{*}=\boldsymbol{\Phi}^{T} \boldsymbol{K} \boldsymbol{\Phi}=\operatorname{diag}\left[\omega_{i}^{2}\right] \\
& \boldsymbol{F}^{*}(t)=\boldsymbol{\Phi}^{T} \boldsymbol{F}(t)
\end{aligned}
$$

For every uncoupled equation, the solution can be given in the form of the convolution integral generally. If transient response of structure is required to be considered in optimization problems, a great deal of computational effort will be involved because the constraints must be satisfied at any time within a certain period of time. It is known that, for every structural candidate in optimization process, the time when the maximal acceleration or the displacement appears can not be known as explicit solution for general excitations, and the accelerations and displacements of all the nodes do not reach their maximal values at the same time. Therefore, the time $t$ must be discretized within the fixed period of time. In this study, since the vibration of engine room structure is caused mainly by the propeller, the excitation is simplified as

$$
\boldsymbol{F}(t)=\overline{\boldsymbol{F}} \times \sin (\bar{\omega} t+\beta)
$$

With this form of excitation, when the vibration reaches the stable state, the stresses of elements and, displacements and accelerations at nodes can be calculated by the mode superposition principle. All quantities vibrate with the frequency of $\bar{\omega}$, and they reach their maximum values simultaneously. These maximum values are independent of the time $t$. As a result, $t$ does not appear in the optimization process.

Eq. 9 can be written separately as below :

$$
\begin{aligned}
& \ddot{q}_{i}+2 \xi_{i} \omega_{i} \dot{q}_{i}+\omega_{i}^{2} q_{i}=\bar{F}^{*}{ }_{i} \sin \left(\bar{\omega}_{t}+\beta\right) \\
& \quad i=1,2, \cdots, p
\end{aligned}
$$

Suppose $\gamma_{i}=\bar{\omega} / \omega_{i}$, the amplitude of normal coordinate $q_{i}$ can be obtained as

$$
A_{i}=\frac{\bar{F}_{i}^{*}}{\omega_{i}^{2} \sqrt{\left(1-\gamma_{i}^{2}\right)^{2}+\left(2 \xi_{i} \gamma_{i}\right)^{2}}}
$$

When the normal coordinates are obtained, the original displacements of nodes can be calculated through Eq. 8. Hence, the dynamic stresses of elements are computed according to the displacements as the same with the static analysis. In vibration control problems, the accelerations of certain points in the structure should be included in the constraints. The accelerations of nodes can be derived from the displacements. Their amplitudes are written as below

$$
|\dot{\boldsymbol{U}}|_{\max }=\bar{\omega}^{2} \boldsymbol{\Phi} \boldsymbol{A}
$$

where

$$
\boldsymbol{A}=\left\{A_{1}, A_{2}, \cdots, A_{p}\right\}^{T}
$$

In this study, damping is taken as the following form :

$$
\boldsymbol{C}=\alpha \boldsymbol{M}+\beta \boldsymbol{K}
$$

therefore

$$
C^{*}=\operatorname{diag}\left(\alpha+\beta \omega_{i}^{2}\right)
$$

from this equation

$$
\xi_{i}=\frac{\alpha}{2 \omega_{i}}+\frac{\beta \omega_{i}}{2}
$$

In ship vibration, $\alpha$ and $\beta$ can take 0.034 and 0.001 respectively.

\section{Penalty Method}

It can be known that the structural optimization problems involve a large number of constraints. In GAs, constraints can be taken account of by penalty methods ${ }^{5), 9)}$ which use penalty functions as an adjustment to the objective function for optimization prob- 
lems. Therefore, a constrained problem is transformed to an unconstrained problem by associating a penalty with any constraint violation. Thus the formula (1) above is transformed into the following function:

$$
F(\boldsymbol{X})=f(\boldsymbol{X})+\sum_{i=1}^{m} \delta_{i} \phi_{i}(\boldsymbol{X})
$$

where $m$ is the total number of constraints, $\delta_{i}$ is the penalty coefficient for constraint $i, \phi_{i}(\boldsymbol{X})$ is a penalty term related to the $i$-th constraint $(i=1, \cdots, m)$.

In the application of the penalty method to the structural optimization problems, generally the static and dynamic constraints represented by the inequalities (2) to (4) are introduced into the new objective function with penalty terms. If the constraints are not satisfied, e. g. the stresses of elements in structure are larger than allowable stresses, or the displacements and accelerations of nodes in structure go beyond the allowable criteria, the candidate for the design of structure will receive penalties. The new function $F(\boldsymbol{X})$ represent the "fitness" of candidate for the design. The penalty terms for this problem can be calculated as the following :

(1) Penalty term of stress constraints

$$
\phi_{s}=\sum_{i=1}^{n e}\left(\frac{\left|\sigma_{i, \max }^{s}\right|+\left|\sigma_{i, \max }^{d}\right|}{\sigma_{i, 0}}-1.0\right)
$$

(2) Penalty term of displacement constraints

$$
\phi_{d}=\sum_{i=1}^{n j}\left(\frac{\left|Y_{j}\right|+\left|U_{j}\right|}{D_{j, 0}}-1.0\right)
$$

(3) Penalty term of acceleration constraints

$$
\phi_{a}=\sum_{i=1}^{n j}\left(\frac{\left|\bar{a}_{j}\right|}{a_{j, 0}}-1.0\right)
$$

The total penalty term can be written as the following form:

$$
\sum_{i=1}^{m} \delta_{i} \phi_{i}(\boldsymbol{X})=\delta_{s} \phi_{s}+\delta_{d} \phi_{d}+\delta_{a} \phi_{a}
$$

Here, $\delta$ is introduced to adjust the weights of the penalties caused by different constraints.

In GAs, the fitness should be positive, therefore the fitness function can be formed as below:

$$
\bar{F}(\boldsymbol{X})=C-F(\boldsymbol{X})
$$

where $C$ is generally a positive constant larger than $F(\boldsymbol{X})$.

\section{Genetic Algorithms}

\section{1 Floating Point Representation}

Reference [6] investigated the applicability of GAs 3),4),9),10) with binary representation of string to the engine room optimization problem under the static stress and dynamic frequency constraints. However for a multidimensional problem with highly precise numerical computation, the searching space becomes too large. Even though the binary alphabet offers the maximum number of schemata per bit of information of any coding, theoretically speaking, the schema theory is not based on only binary alphabets. The experiment ${ }^{3)}$ with large alphabets has shown that the real coding technique has worked well in a number of practical problems. By using the floating point representation for the string of solution, the chromosome vector has the same length with the solution vector. In the binary implementation, each element of a chromosome vector is coded using the same number of bits. To preserve the precision requirement, the chromosome vector is much larger than the solution vector. As a result, by using the floating point representation, the computational storage can be saved significantly and the computational time can be also reduced because there is no need to decode the binary representation to the real number. The precision of the binary approach depends on the number of bits, but for the floating point approach, it depends on the underlying machine and is generally much better than that of binary representation. The GAs can directly work on coding space and solution space alternatively. Since the floating point representation has the nature that two points close to each other in the representation space must also be close in the problem space, and vice visa, it is easier to design some new operators. In this research, some of them are examined in order to investigate their effects on the performance in the GA.

\section{2 Operators of the Genetic Algorithm}

In addition to the elitism, simple crossover and uniform mutation, which are commonly included in practical applications ${ }^{1,2), 10)}$, the operators of arithmetic crossover, boundary mutation and non-uniform mutation are introduced in this calculation.

The operator of simple crossover here works in the same way as that in GAs with binary representation. For the structural optimization problems, there exists no possibility for the crossed offspring exceeding the domain constraints. The parents exchange their genes at a random position simply.

The operator of arithmetical crossover is defined as a linear combination of two vectors through a random number $\alpha$ described by Fig. 1. The introduction of this operator is based on the understanding that the better point may exists on the line connecting the two good points.

The operator of uniform mutation randomly changes one or a few design variables directly with a random value with uniform probability distribution.

The operator of non-uniform mutation is responsible for the fine tuning capabilities of the solution. When GAs proceed to the later generations, the force driving the solution to the optimum point becomes quite small.

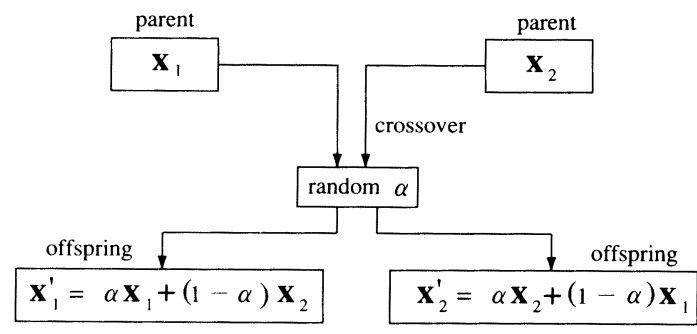

Fig. 1 Arithmetic Crossover 
In fact, the optimum point is very near the best point obtained at the present generation. This operator changes the "best" point a little at the later generations to speed up the convergence of the GA. It works as follows. Suppose variable $x_{k}$ is selected for this mutation, the result of this mutation is:

$$
x_{k}^{\prime}=\left\{\begin{array}{l}
x_{k}+\Delta\left(t, \operatorname{right}(k)-x_{k}\right) \text { if } \gamma=0 \\
x_{k}+\Delta\left(t, x_{k}-\operatorname{left}(k)\right) \text { if } \gamma=1
\end{array}\right.
$$

where $\gamma$ is a random binary digit, $t$ denotes the number of generation, $\operatorname{right}(k)$ and left $(k)$ are the right bound and left bound of $x_{k}$. Function $\Delta(t, y)$ is taken as the following form :

$$
\Delta(t, y)=y \cdot r \cdot\left(1-\frac{t}{T}\right)^{b}
$$

where $r$ is a random number from 0 to $1, T$ is the maximal generation number, and $b$ is the coefficient for this mutation determining the degree of non-uniformity. In this study, $b=2$.

Since the optimal solution in structural optimizations lies often on or near the boundary of the constraints, it is essential to introduce the operator of boundary mutation to hasten the rate of convergence. With this mutation, the mutated $x_{k}$ is changed to be either right bound value or left bound value with equal probability.

\section{3 Optimization Procedure}

Fig. 2 shows the procedure of the optimization used in this research. Classical GAs operate on binary strings, however here the floating point representation for strings together with some operators developed for it are introduced. In classical GAs, the reproduction is implemented by proportional selection. Here the elitism is introduced to improve the efficiency of convergence. The constraints on dynamic response are includ-

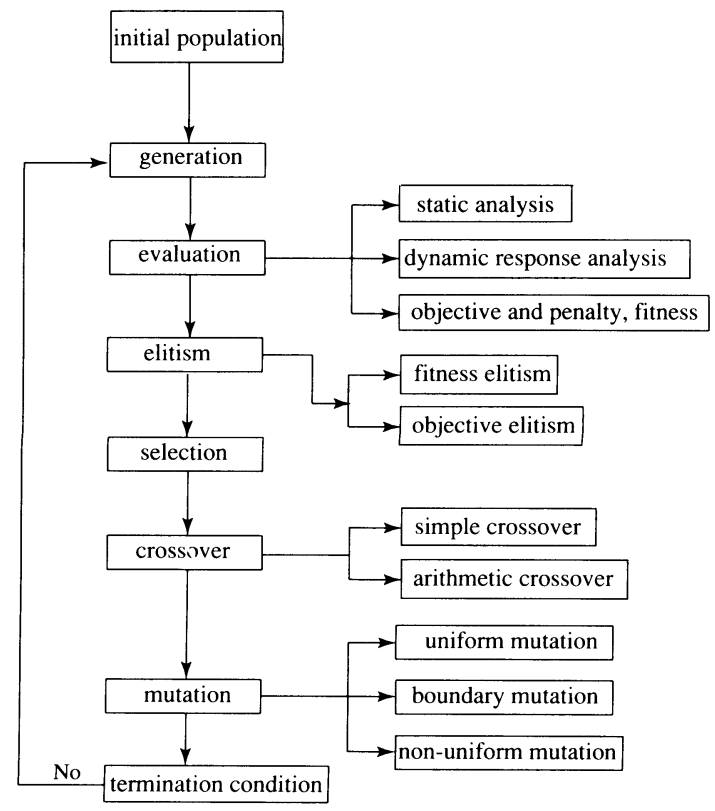

Fig. 2 Optimization Procedure ed in this study. Addition to the operator of simple crossover used in classical GAs, the arithmetic crossover is also applied. For the mutation, the operators of boundary mutation and non-uniform mutation are employed and investigated.

\section{Numerical Example}

\section{1 Computing Model}

The principal dimensions of the ship for numerical example are:

Length $\mathrm{P} . \mathrm{P}=70 \mathrm{~m}$; Breadth (mld.) $=12 \mathrm{~m}$;

Depth $(\mathrm{mld})=.7.12 \mathrm{~m}$; Draught $=4.14 \mathrm{~m}$;

Weight of main engine $=31$ tonf ;

Weight of generator $=2$ tonf $\times 2$ sets;

$\mathrm{SHP}=1800$ PS ; Blade number $=4$;

Propeller $D_{p}=2.40 \mathrm{~m} ; N=284 \mathrm{rpm}$.

The simplified three-dimensional finite element model of the engine room part is shown in Fig. 3. The virtual added water mass is considered in the dynamic analysis. The numbers of elements and nodal points used in this model are 156 and 98 respectively.

The propeller excitation is assumed to be applied to the rear of the ship in vertical direction as shown in Fig. 3. Since it is difficult to obtain the reliable data for all the forces caused by the propeller, only the surface force in vertical direction is estimated according to the following formula ${ }^{11), 12)}$

$$
F_{z s}=\frac{K_{s} \cdot S H P}{(Z-1)\left(C / D_{p}\right)^{3 / 2} \cdot N \cdot D_{p}}
$$

where $K_{s}=0.031, C / D_{p}=0.25$.

The propeller with four blades is attached to the main engine whose revolution is $284 \mathrm{rpm}$, resulting in the excitation frequency of $18.933 \mathrm{HZ}$. The static loading condition is shown in Fig. 4. The uniform loads $q_{1}(0.5$ $\mathrm{m}$ water head) and $q_{2}\left(150 \mathrm{kgf} / \mathrm{m}^{2}\right)$ are applied to the upper deck and the second deck, respectively. The allowable bending stress and the acceleration are 18 $\mathrm{kgf} / \mathrm{mm}^{2}$ and 40 gal respectively.

Design variables include the sizes of cross-section of deck web beams $(A-A)$, ship side web frames $(B-B)$ and bottom floors $\left(\mathrm{C}^{--} \mathrm{C}\right)$ as shown in Fig. 4. The cross -sections of all the deck web beams take the same

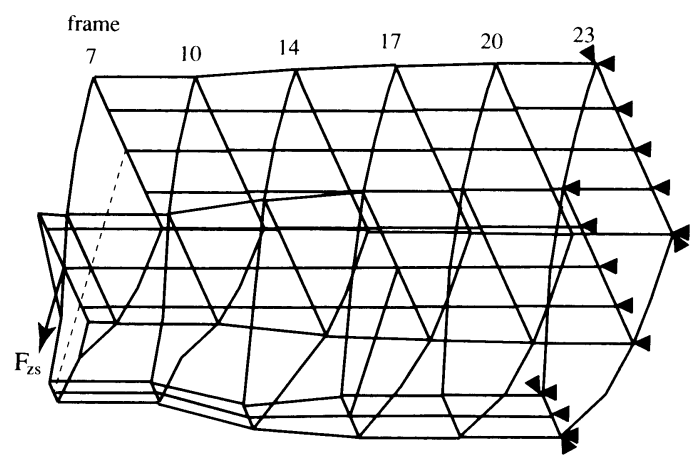

Fig. 3 Structural Model 


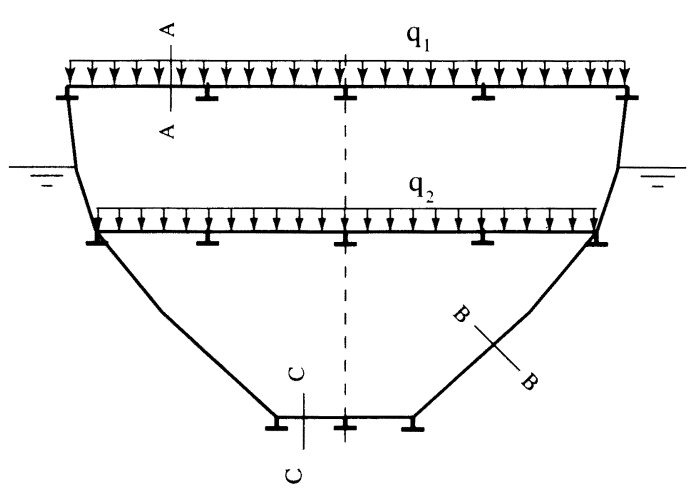

Static Load

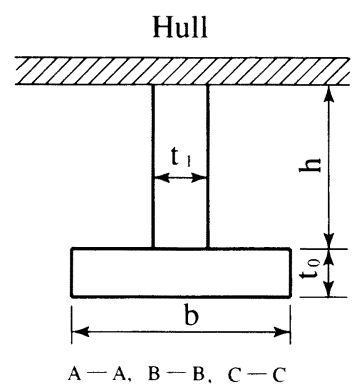

Design Variables:

\begin{tabular}{|c|c|c|}
\hline deck & side & bottom \\
\hline $\mathrm{h}_{\mathrm{A}}$ & $\mathrm{h}_{\mathrm{B}}$ & $\mathrm{h}_{\mathrm{C}}$ \\
\hline $\mathrm{t}_{0 \mathrm{~A}}$ & $\mathrm{t}_{0 \mathrm{~B}}$ & $\mathrm{t}_{0 \mathrm{C}}$ \\
\hline $\mathrm{t}_{1 \mathrm{~A}}$ & $\mathrm{t}_{1 \mathrm{~B}}$ & $\mathrm{t}_{1 \mathrm{C}}$ \\
\hline $\mathrm{b}_{\mathrm{A}}$ & $\mathrm{b}_{\mathrm{B}}$ & $\mathrm{b}_{\mathrm{C}}$ \\
\hline
\end{tabular}

Fig. 4 Static Loading and Design Variables

values. The ship side web frames and bottom floors are handled in the same way, therefore there are only twelve design variables in this problem. The bounds of these design variables are listed in Table 1 . For all the members, the bound of each size of cross-section is assumed to be the same.

\section{2 Optimization Results}

Even though the design variables vary with generations, the low-order eigenmode shapes, which are shown in Fig. 5, are almost the same. It is observed that mode 3 and mode 5 do not make any contribution to the structural response because both the structure and the excitation are symmetric. Mode 4 exhibits the local vibration of the deck. For mode 1 and mode 2, not only local vibration but also global vibration are observed in the first and second decks, while the bottom of ship undergoes the global vibration mainly.

The design variables of optimum design of the structure and the corresponding areas and moments of inertia of areas are provided in Table 3 . It can be seen clearly that almost all web thickness of stiffeners $\left(t_{1}\right)$ reach their bounds because they have strongest force of driving the GA process to the optimum point. The moment of inertia of area of a member $I_{y}$ is proportional to $h^{3}$ ( $h$ is the web height of stiffeners), however the area of its cross ${ }^{-}$section is only proportional to $h$. In order to increase the stiffness under the condition of diminishing the area of the cross-section, the efficient way is to increase the web height and decrease the web thickness simultaneously. This process is repeated until
Table 1 Bound of design variables

\begin{tabular}{|c|c|c|c|c|}
\hline variable & $\mathrm{h}$ & $\mathrm{t}_{0}$ & $\mathrm{t}_{1}$ & $\mathrm{~b}$ \\
\hline $\begin{array}{c}\text { bound } \\
(\mathrm{mm})\end{array}$ & $200 \sim 600$ & $8 \sim 20$ & $8 \sim 16$ & $60 \sim 200$ \\
\hline
\end{tabular}
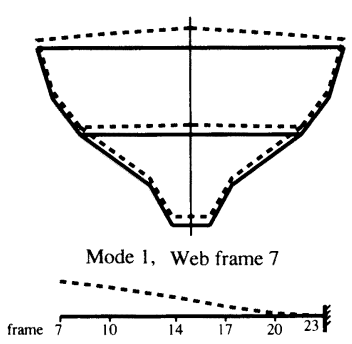

Mode 1, Center girder of upper deck

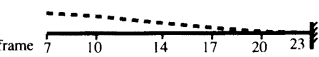

Mode 1, Engine girder

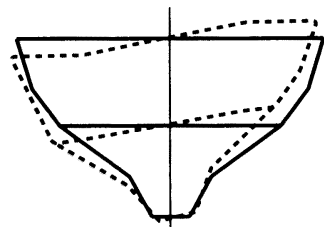

Mode 3, Web frame 7

Mode 5, Web frame 7

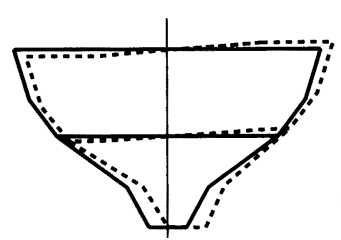

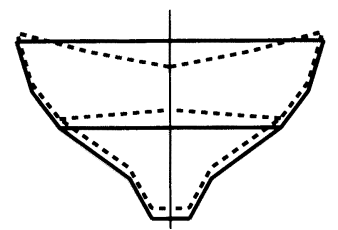

Mode 2, Web frame 7

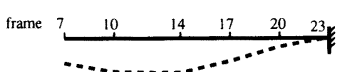

Mode 2, Center girder of upper deck

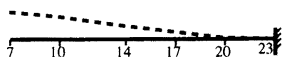

Mode 2, Engine girder

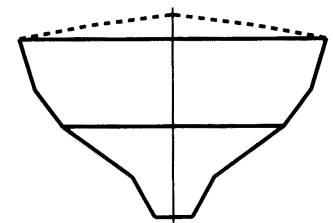

Mode 4, Web frame 7

Mode 4, Center girder of upper deck

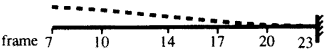

Mode 5, Center girder of upper deck in horizontal direction

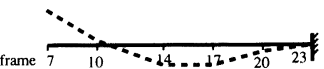

Fig. 5 Eigenmode Shape

Table 2 Results of Design Variables after the First Generation

\begin{tabular}{|c|c|c|c|c|c|c|c|c|}
\hline & $\mathrm{h}$ & $\mathrm{t}_{0}$ & $\mathrm{t}_{1}$ & $\mathrm{~b}$ & $\mathrm{~S}$ & $\mathrm{I}_{\mathrm{p}}(10)$ & $\mathrm{I}_{\mathbf{y}}\left(10^{3}\right)$ & $\mathrm{I}_{\mathbf{z}}\left(10^{5}\right)$ \\
\hline deck & 210.8 & 14.8 & 9.9 & 190.2 & 15709 & 63530 & 126581 & 10583 \\
\hline side & 349.2 & 18.3 & 12 & 117.7 & 17138 & 77663 & 324448 & 10523 \\
\hline bottom & 558.0 & 13.9 & 14.4 & 91.36 & 12906 & 74960 & 568710 & 399.0 \\
\hline
\end{tabular}

Table 3 Optimization Results of Design Variables

\begin{tabular}{|c|c|c|c|c|c|c|c|c|}
\hline & $\mathrm{h}$ & $\mathrm{t}_{0}$ & $\mathrm{t}_{1}$ & $\mathrm{~b}$ & $\mathrm{~S}$ & $\mathrm{I}_{\mathrm{p}}(10)$ & $\mathrm{I}_{\mathbf{y}}\left(10^{3}\right)$ & $\mathrm{I}_{\mathrm{z}}\left(10^{5}\right)$ \\
\hline deck & 200.0 & 11.9 & 8.00 & 137.6 & 14034 & 47093 & 77049 & 10523 \\
\hline side & 502.6 & 8.19 & 8.03 & 67.70 & 15389 & 45811 & 382382 & 10500 \\
\hline bottom & 308.0 & 16.1 & 8.00 & 94.40 & 7616 & 29256 & 137790 & 401.0 \\
\hline
\end{tabular}


their web thickness reach the lower bound and the web height of the cross $^{-}$section reaches the larger value of $502.6 \mathrm{~mm}$ for the side web frame. Because the load on the deck beams is small and the length of transverse web frames in bottom is short, the web thickness of stiffeners $\left(t_{1}\right)$ in deck web beam and bottom floor reach their bounds while their web heights $(h)$ decrease to 200 $\mathrm{mm}$ and $308 \mathrm{~mm}$ respectively. Together with Table 2 which gives the same items of the best chromosome in the first generation, it can be observed that the areas $(S)$ of the cross-sections of the optimized members decrease significantly, while the moments of inertia of areas $I_{y}$ of the side web frames increase. The stiffness of these members influences the static and dynamic response more than the web frames in bottom floor because the lengths of the transverse members in bottom are small. The polar moments of inertia expressed by $I_{p}$ decrease because they, together with the longitudinal members, contribute mainly to the global torque and lateral response which does not appear in this computing model. The lateral moments of inertia of areas denoted by $I_{z}$ change very little because the widths of the associated effective plating are large. It seems that the GA can find the important variable to be changed during the first a few generations. For this case, decreasing the web thickness of stiffeners $\left(t_{1}\right)$ is the most efficient to decrease the area of the cross $^{-}$ section because the web height is quite large. Increasing the web height $h$ is also the good direction to increase the moment of inertia of area because it is proportional to the third power of the web height $\left(h^{3}\right)$.

The results of the structural analyses for the solutions of the first and final generations are shown in Table 4. As discussed above, mode 3 and mode 5 do not make any contribution to the dynamic response for this problem. Therefore the key issue is to make the frequencies of mode 2 and mode 4 avoid the excitation frequency of $119 \mathrm{rad} / \mathrm{s}$. It is obvious that $\omega_{4}$ of the first generation is closer to the excitation frequency than the one of the final optimal design. Even though the solution of the first generation meets all the requirements, it is not the optimal solution. The value of objective function is much larger than the optimal value. From the optimal results, it can be known that all the natural frequencies smaller than $119 \mathrm{rad} / \mathrm{s}$ become smaller and the larger ones become larger. $\omega_{4}$ reaches the value $111.50 \mathrm{rad} / \mathrm{s}$ so that the acceleration of the structure approaches the edge of the constraints, which is 40 gal for this example.

The maximal acceleration of this structure at the

Table 4 Comparison between the first and final generation

\begin{tabular}{|c|c|c|c|c|c|c|c|c|}
\hline generation & $\omega_{1}$ & $\omega_{2}$ & $\omega_{3}$ & $\omega_{4}$ & $\omega_{5}$ & $\begin{array}{c}\mathrm{a}^{\mathrm{m}} \\
(\mathrm{gal})\end{array}$ & $\begin{array}{c}\sigma^{\mathrm{m}} \\
\left(\mathrm{kg}^{\mathrm{m}} / \mathrm{mm}^{2}\right)\end{array}$ & obj (kg) \\
\hline first & 58.28 & 87.42 & 99.97 & 113.75 & 132.58 & 38.09 & 16.63 & 6839.75 \\
\hline final & 53.97 & 84.06 & 99.72 & 111.50 & 135.36 & 39.81 & 17.81 & 4631.17 \\
\hline
\end{tabular}

final optimization result is shown in Fig. 6. It is known that the maximal acceleration happens at the stern part of ship where the external excitation is exerted. Accordingly, the acceleration constraints can be imposed only upon the nodal points of web frames 7 to save the computing time. It is noticed that the maximal acceleration reaches almost the allowable value at the bottom. The acceleration constraints are active in this problem. The maximal stresses are $16.63 \mathrm{kgf} / \mathrm{mm}^{2}$ and $17.81 \mathrm{kgf} / \mathrm{mm}^{2}$ in the best candidates for the designs at the first generation and in the optimal design, respectively. The former stress happens at the lower part of side web frame 14, while the latter stress appears at the both ends of the deck beam of frame 10 on the first floor.

The progress of the optimization with all the operators mentioned above is shown in Fig. 7. Here pop-size $=30$, the probability of uniform mutation $p_{s m}$ is 0.01 , the probability of non uniform mutation $p_{n m}$ is 0.01 , the coefficient $b=2$, the probability of boundary mutation $p_{b m}$ is 0.01 , the probability of simple crossover $p_{a c}$ is 0.70 , the probability of arithmetic crossover $p_{a c}$ is 0.1 . The process terminates at about generation 120. The original objective function, weight of structure, decreases quickly within the first a few generations. Between the 10th and 55th generation, the convergence becomes quite slow.

\section{3 Floating Point Representation}

There are 12 design variables in this numerical prob-
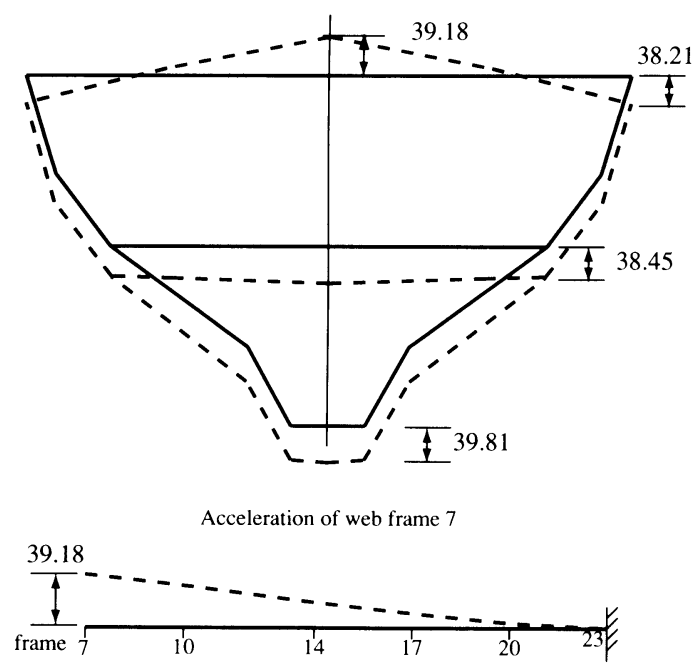

Acceleration of center girder of upper deck

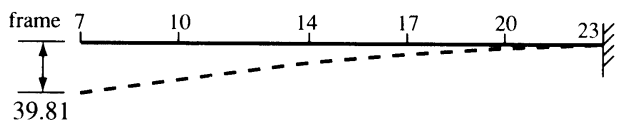

Acceleration of engine girder

Fig. 6 Amplitude of Acceleration 


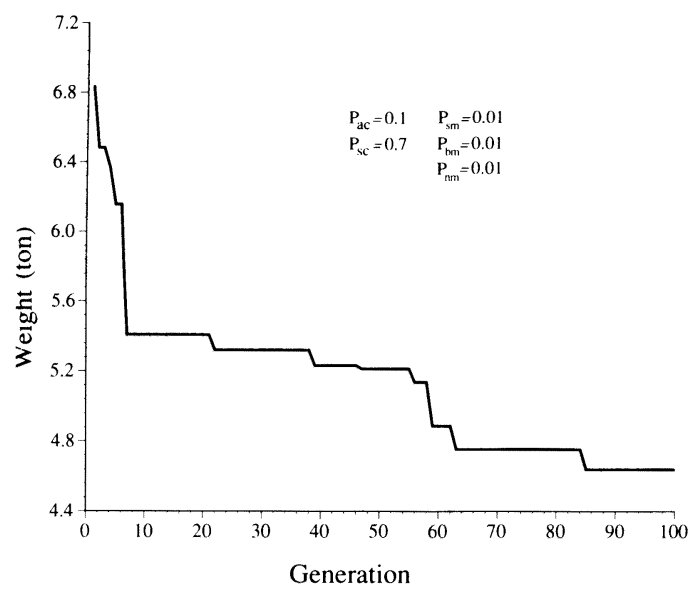

Fig. 7 Progress of the GA

lem. If the binary representation to the string of solution is applied with the precision of millimeter for design variables, the length of the binary solution vector becomes 111. However for the floating point representation, the length of the solution vector is only 12. Moreover, the precision of the solution based on the floating point representation is much higher than the binary representation.

\section{4 Influences of Operators}

\section{4. 1 Probability of Crossover}

Fig. 8 shows the results of the optimization with different $p_{s c}$. The other GA parameters are the same as before. It is observed that, at the first stage of the evolution process, the smaller the $p_{s c}$ is, the faster the convergence of the process is, generally. However when $p_{s c}=0.1$, the process terminates prematurely. Even though the process with $p_{s c}=0.7$ converges slowly at first compared with the one with $p_{s c}=0.4$, it reaches the optimum faster slightly.

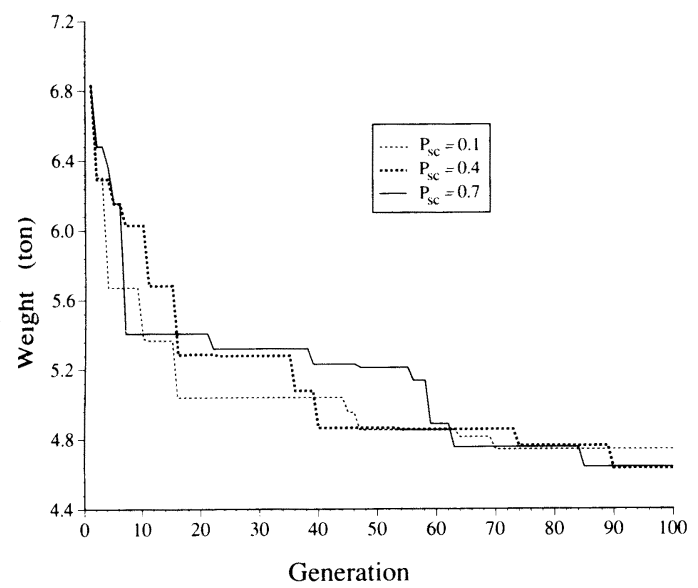

Fig. 8 Influence of Probability of Crossover

\section{4.2 Probability of Mutation}

The results of the optimization with different probability of mutation are given in Fig. 9. Here pop-size= 50, $p_{n m}=0, p_{b m}=0, p_{s c}=0.70 . p_{a c}=0.1$. It is noticed that when $p_{s m}$ is too small, the optimization converges rapidly and prematurely. However large $p_{s m}$ may slow the process to the optimum.

\section{4. 3 Arithmetic Crossover}

The result of Simple GA is carried out by introducing only the operators of simple crossover and uniform mutation whose probabilities are 0.8 and 0.02 respectively. In the GA with arithmetic crossover, $p_{s c}=0.6$, $p_{a c}=0.2, p_{m}=0.02$. From Fig. 10 , it can be observed that both of them converge to the optimum and the one with arithmetic crossover converges faster slightly. For Simple GA, a horizontal line, which means that no improvement is observed in the objective function, between generation 20 and 50 appears. If this line is too long, the false appearance of convergence may happen which may result in premature. However it seems that the arithmetic crossover can avoid this problem.

6. 4. 4 Boundary Mutation

The dotted line in Fig. 11 shows the result of GA with boundary mutation, where $p_{b m}=0.01$ and the other

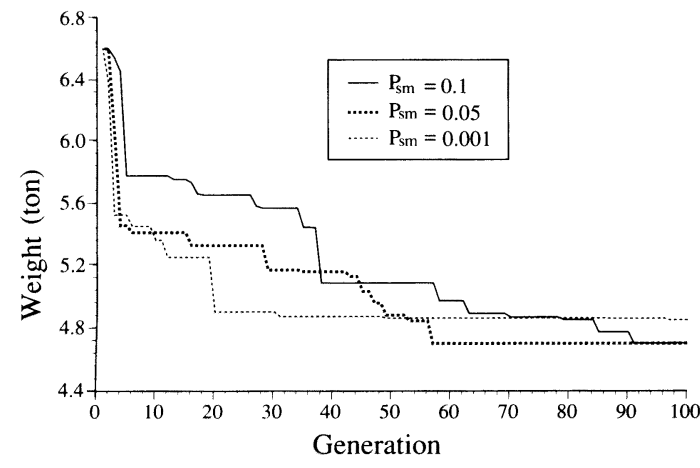

Fig. 9 Influence of Probability of Mutation

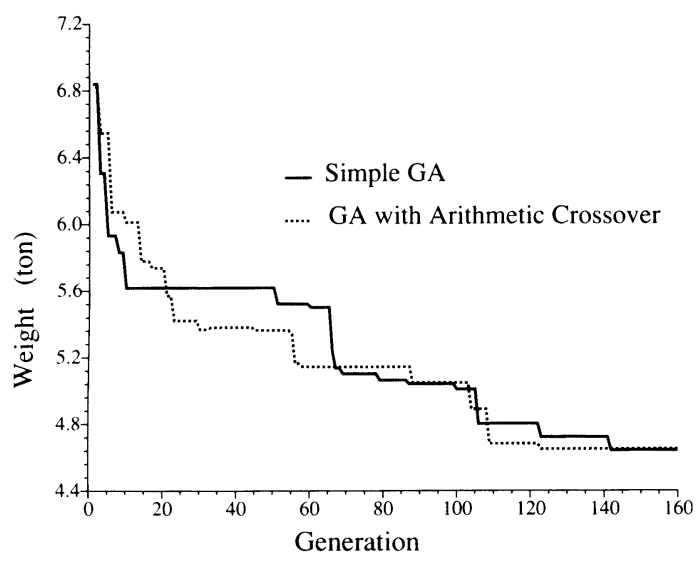

Fig. 10 Influence of Arithmetic Crossover 


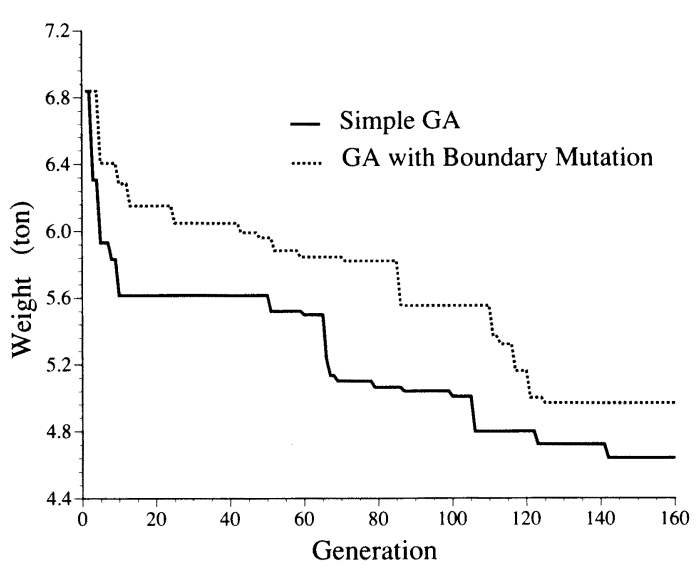

Fig. 11 Influence of Boundary Mutation

parameters are the same as the ones of Simple GA. It is noticed that the GA with boundary mutation can not find the optimum for this example problem. It appears that there is no good effect on the optimization process if only the operator of boundary mutation is introduced. It may be useful for this operator to be applied to GA together with other operators such as the operators of arithmetic crossover and non-uniform mutation.

\section{4.5 Non-uniform Mutation}

The result of GA with non-uniform mutation is given by the dotted curve of Fig. 12. The probability of non -uniform mutation is 0.01 and the other parameters are the same as the ones of Simple GA. It can be seen clearly that the solution is improved little by little with the progress of the generations. With the introduction of this operator, it seems that the false convergence can be avoided. It converges slower than Simple GA at first, but reaches the optimum faster.

\section{4.6 A Set of Operators}

It is difficult to say how much of the probability of

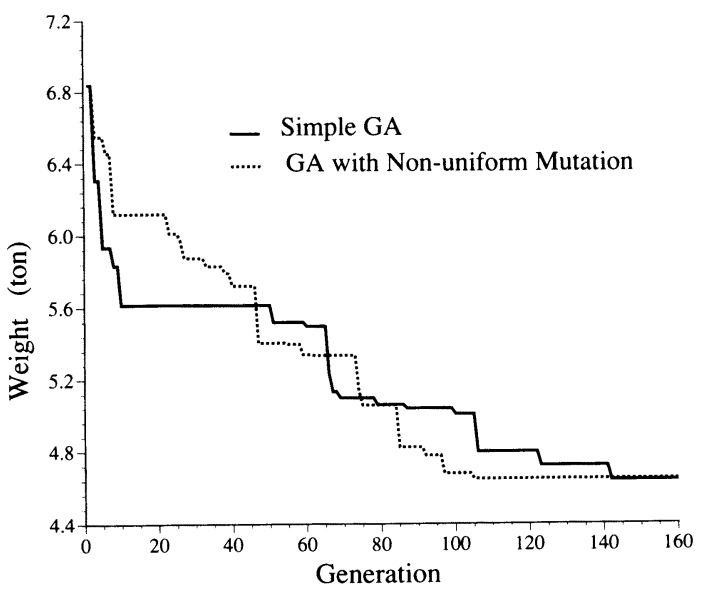

Fig. 12 Influence of Non-uniform Mutation every operator is the best for convergence of the evolutionary process since all the operators influence each other. For a specific problem, there must be an optimal set of operators which can make the process of optimization for the problem reach the optimum with the smallest number of generations. However, finding the optimal set of operators itself is a very difficult optimization problem. It seems that the best set of operators is problem-dependent. For this numerical example, the low $p_{c}(=0.2)$ and high $p_{m}(=0.25)$ have been used and the optimum was obtained also. Since there are several uncertainties on the combination of operators, further experiments are required.

\section{Conclusions}

1) The Genetic Algorithm was applied to structural optimization of engine room of ship considering the static and dynamic response constraints successfully. The result is more reliable than that with the frequency constraints. The acceleration or displacements of structure can be controled.

2) The floating point representation to the string of solution can save more computing storage than the binary one. The precision of the solution is enough for engineering problems.

3) Large probabilities of crossover and mutation may slow the convergence of GAs. However if they are too small, it may result in the premature.

4) The operators of arithmetic crossover, non-uniform mutation and boundary mutation make the optimization more effective if they are applied together. It seems that the arithmetic crossover and non-uniform mutation can keep the GA process away from the premature. The non-uniform mutation is useful for the fine tuning of the solution. The operator of boundary mutation can speed up the convergence of optimization process for general engineering problems theoretically and is used in this calculation. However using only this operator may lead the GA process to premature.

\section{References}

1) Tetsuo Okada, Isao Neki, Optimization of Ship Structural Design by Genetic Algorithm, J. Soc. Naval Arch. of Japan, (April, 1992), pp 259-266.

2) Isao Neki, Tetsuo Okada, Optimum Structural Design Using Genetic Algorithm and Finite Element Method, J. Soc. Naval Arch. of Japan, (July, 1995) pp 327-337.

3) Zbigniew Michalewicz, Genetic Algorithms + Data Structures=Evolution Programs, 3rd rev. Springer-Verlag. 1996.

4) Goldberg, D. E., Genetic Algorithms in Search, Optimization and Machine Learning, Addison Wesley, Reading, Mass., 1989.

5) Guoqiang Zhou, Hisashi Nobukawa and Fengxiang Yang, Discrete Optimization of Ship Structures from the Viewpoint of Practical Design, J. Soc. Naval Arch. of Japan, (Nov. 1997), pp 551559. 
6) Hisashi Nobukawa, Yang Fengxiang, Mitsuru Kitamura, Zhou Guoqiang, Optimization of Engine Room Structure under Static and Dynamic Constraints Using Genetic Algorithms. J. Soc. Naval Arch. of Japan, (May. 1998), pp 315 $-322$.

7) Jeffrey A. Joines, Christopher R. Houck, On the Use of Non-stationary Penalty Functions to Solve Nonlinear Constrained Optimization Problems with GA's. IEEE (1985), pp 579-584.

8) Michalewicz, Z. and Janikow, C., Handling Constraints in Genetic Algorithms, Proceeding of the Fourth International Conference on Genetic Algorithms, (1991), pp 151-157.
9) Mitsuo Gen, Runwei Cheng, Genetic Algorithms and Engineering Design, JOHN WILEY \& SONS, INC. 1997.

10) Hisashi Nobukawa, Guoqiang Zhou, Discrete Optimization of Ship Structures with Genetic Algorithms, Journal of The Society of Naval Architects of Japan, Vol. 179, (1996), pp 293-301.

11) Kaname Taniguchi, Oscillating Pressure near Propellers, Trans. West-Japan Society of Naval Architects, Vol. 16, (1958), pp 19-30 (in Japanese) .

12) Technological Research Report of West-Japan Society of Naval Architects, Vol.11, (1971) (in Japanese). 